\title{
COMPARING WILD 2 PARTICLES TO CHONDRITES AND IDPS
}

Michael Zolensky

Astromaterials Research and Exploration Science, NASA Johnson Space Center, Houston, Texas 77058, USA

michael.e.zolensky@nasa.gov

Keiko Nakamura-Messenger

Jacobs Sverdrup, ESCG, Houston, TX 77058, USA

Frans Rietmeijer

Department of Earth and Planetary Sciences, University of New Mexico, MSC 03-2040, Albuquerque, NM 87131-0001, USA

Hugues Leroux

Laboratoire de Structure et Propriétés de l'Etat Solide, Bat C6, Université des Sciences et Technologies de Lille, 59655 Villeneuve d'Ascq, France

Takashi Mikouchi

Department of Earth and Planetary Science, University of Tokyo, Hongo, Bunkyo-ku, Tokyo 113-0033, Japan

Kazumasa Ohsumi

Institute of Materials Structure Science, Tsukuba-shi, Ibaraki-ken, 305, Japan

Steven Simon

Department of the Geophysical Sciences, The University of Chicago, 5734 S. Ellis Ave., Chicago, IL 60637 USA

Lawrence Grossman

Department of the Geophysical Sciences, The University of Chicago, 5734 S. Ellis Ave., Chicago, IL 60637 USA and

The Enrico Fermi Institute

The University of Chicago, 5640 S. Ellis Ave., Chicago, IL 60637, USA

Thomas Stephan

Institut für Planetologie, Westfälische Wilhelms-Universität Münster, Wilhelm-Klemm-Str.10, 48149 Münster, Germany

Michael Weisberg

Physical Sciences, Kingsborough Community College (CUNY), Brooklyn, NY 11235, USA

Michael Velbel

Department of Geological Sciences, 206 Natural Science Building, Michigan State University, East Lansing, MI 48824-1115, USA 
Thomas Zega and Rhonda Stroud

Code 6360, Naval Research Laboratory, 4555 Overlook Ave. SW, Washington, DC 20375, USA

Kazushige Tomeoka, Ichiro Ohnishi, and Naotaka Tomioka

Department of Earth and Planetary Sciences, Faculty of Science, Kobe University, Nada, Kobe 657-8501, Japan

Tomoki Nakamura

Department of Earth and Planetary Sciences Faculty of Sciences Kyushu University Hakozaki, Fukuoka 812-8581, Japan

Graciela Matrajt, David Joswiak and Don Brownlee

Department of Astronomy, University of Washington, Seattle, WA 98195, USA

Falko Langenhorst

Institute of Geosciences, Friedrich-Schiller-University Jena, Burgweg 11, D-07749 Jena, Germany

Alexander Krot

Hawaii Institute of Geophysics and Planetology, University of Hawaii, Honolulu, HI 96822, USA

Anton Kearsley

Department of Mineralogy, Natural History Museum, Cromwell Road, London, SW7 5BD, UK

Hope Ishii, Giles Graham, Zu Rong Dai, Miaofang Chi and John Bradley

Institute for Geophysics and Planetary Physics, Lawrence Livermore National Laboratory, Livermore, CA 94550, USA

Kenji Hagiya

Graduate School of Life Science, University of Hyogo, Koto 3-2-1, Kamigori, Ako-gun, Hyogo 678-1297, Japan

Matthieu Gounelle

Museum National d'Histoire Naturelle, Laboratoire d'Etude de la Matiere Extraterrestre, USM 0205 (LEME), Case Postale 52, 57, rue Cuvier, 75005 Paris, France

John Bridges

Planetary and Space Sciences Research Institute, Open University, Milton Keynes, MK7 6AA, UK

Resubmitted to MAPS December, 2007 


\begin{abstract}
We compare the observed composition ranges of olivine, pyroxene and Fe-Ni sulfides in Wild 2 grains, comparing these with chondritic IDPs and chondrite classes to explore whether these data suggest affinities to known hydrous materials in particular. Wild 2 olivine has an extremely wide composition range, from $\mathrm{Fo}_{4-100}$ with a pronounced frequency peak at Fo99. The composition range displayed by the low-calcium pyroxene is also very extensive, from $\mathrm{En}_{52}$ to $\mathrm{En}_{100}$, with a significant frequency peak centered at $\mathrm{En}_{95}$. These ranges are as broad or broader than those reported for any other extraterrestrial material. Wild $2 \mathrm{Fe}-\mathrm{Ni}$ sulfides mainly have compositions close to that of $\mathrm{FeS}$, with less than 2 atom $\% \mathrm{Ni}$ - to date, only two pentlandite grains have been found among the Wild-grains suggesting that this mineral is not abundant. The complete lack of compositions between FeS and pentlandite (with intermediate solid solution compositions) suggests (but does not require) that $\mathrm{FeS}$ and pentlandite condensed as crystalline species, i.e. did not form as amorphous phases, which later became annealed. While we have not yet observed any direct evidence of water-bearing minerals, the presence of Ni-bearing sulfides, and magnesium-dominated olivine and low-Ca pyroxene does not rule out their presence at low abundance. We do conclude that modern major and minor element compositions of chondrite matrix and IDPs are needed.
\end{abstract}

\title{
Introduction
}

The nature of cometary solids is of fundamental importance to our understanding of the early solar nebula and protoplanetary history. Until now we have had to study comets from afar using spectroscopy, or settle for analyses of interplanetary dust particles (IDPs) of uncertain provenance. The community had long suspected that the anhydrous chondritic IDPs included cometary particles, but since the arguments were partly statistical in nature it was impossible to determine this for any particular particle (Nier and Schlutter, 1990, 1992, 1993). However, thanks to the Stardust mission, we can now perform mineralogical and petrographic analyses of particles derived directly from the Jupiter-family Comet 81P/Wild 2.

For the first year that Wild 2 samples were available we worked very hard to perform strictly preliminary investigations of many aspects of the samples, including mineralogy and petrology of the captured particles This PET period has now ended and we are now entering an extended phase of in-depth investigations.

The apparent complete lack of aqueous alteration products in Wild 2 samples is in clear contrast to the mineralogy reported for Comet Tempel 1, based upon Spitzer Space Observatory data in support of the Deep Impact Mission (Lisse et al., 2006; 2007). This mineralogical difference could be due to differences in the geological histories of Jupiter-family comets. Future petrological studies of Wild 2 dust will be needed before would with any degree of confidence disclaim the presence of layer silicates in this comet. The presence of layer silicates in some of the chondritic porous IDPs (Rietmeijer, 1998) suggests that these minerals might be found among the collected Wild 2 dust. In this paper we compare the observed composition ranges of olivine, pyroxene and Fe-Ni sulfides in Wild 2 grains, comparing these with chondritic IDPs and chondrite classes, and explore whether these data suggest affinities to known hydrous materials in particular.

\section{Experimental procedure}


To remove grains from aerogel we used a combination of old and new techniques. For larger features we used straight razor blades to trim away excess aerogel. For the bulk of the tracks however, we mainly used two newly-developed techniques: keystoning and quikstoning.

Whole tracks in the aerogel collectors were extracted using the keystoning technique developed at the Space Sciences Laboratory, the University of California at Berkeley (Westphal et al, 2004). Glass rods (1 $\mathrm{mm}$ diameter) are pulled to make two microneedles. The needles are held by micromanipulators which are attached to the stage of the extraction microscope. The needles cut the aerogel by repetitive "poking" (up to 4 million pokes per extracted track). The micromanipulators are driven automatically by computer. First, an angled cut is made which undercuts the deepest feature of a particular impact; then a vertical cut is made around the impact. The resulting wedge-shaped block of aerogel (a "keystone") contains the entire impact track and the terminal particles. The keystone is then removed from the collector using silicon microforks which are inserted into pre-machined holes in the keystone. To extract grains from the keystone, it can be flattened between two sheets of Mylar, and the grains can be easily separated. The flattened keystone preserves the general positions of the enclosed grains, permitting surveys to be made of mineralogical variation down tracks. For Stardust samples, we made keystones at the University of California, Berkeley, and at the NASA Johnson Space Center (JSC).

In quikstoning, a diamond, steel or sapphire utility-knife-shaped blade is driven through the aerogel at ultrasonic frequencies. This micro-blade is controlled by a micromanipulator for fine motion control. The ultrasonic oscillations are generated by the piezo-driver of a MicroDissector (Eppendorf) which is mounted on the micromanipulator. Details of the instrument are given by Ishii et al., 2005. Quikstoning for this work was performed at the Lawrence Livermore National Lab and at NASA JSC.

The embedding media for Wild 2 grains were EMBED-812 epoxy, sulfur and WELD-ON 40 acrylic. We most frequently used acrylic to mount grains for ultramicrotomy. Particles could then be easily removed from the embedding medium using common organic solvents, including acetone or chloroform, permitting subsequent isotopic or bulk composition analyses. One problem we encountered was that acrylic polymerized in an electron beam, making subsequent grain removal difficult. We embedded pieces of aerogel in EMBED-812 epoxy, during which the aerogel became completely invisible, revealing all of the grains in a track in the most complete manner.

When it was necessary to avoid carbon contamination, Comet Wild 2 grains were also embedded in high-purity $\mathrm{S}$ and sliced into 50 $70 \mathrm{~nm}$-thick sections with an ultramicrotome equipped with a diamond knife. The sections were floated onto ultra-pure water and transferred to amorphous $\mathrm{C}$-supported $\mathrm{Cu}$ Transmission Electron Microscopy (TEM) grids. The $\mathrm{S}$ was sublimed prior to analysis focused on organic matter in the sample such as C- and N- X-ray Absorption Near-Edge Structure (XANES), Fourier Transform Infra-Red spectroscopy (FTIR) and light element isotopic analysis in the Nano- Secondary Ion Mass Spectrometer (NanoSIMS). Sulfur was chosen as an embedding medium to avoid contamination of the samples with low viscosity resin (epoxy) normally used for ultramicrotomy. Sulfur beads containing the samples were attached to a sample holding bullet using cyanoacrylate. To evaluate the potential glue contribution to the sample analysis, sulfur beads devoid of sample were prepared in the same manner. We did not see any evidence that cyanoacrylate penetrated the $\mathrm{S}$ bead during subsequent TEM investigation of the sample-free S slices. Electron Energy-Loss Spectroscopy (EELS) spectra acquired from the $\mathrm{S}$ test slices also did not show evidence of the pronounced $\mathrm{CN}$ peak characteristic of cyanoacrylate. 
Micro-FTIR was used to provide a rapid, non-destructive pre-characterization of most grains removed from the aerogel at JSC. This technique was most valuable for terminal grains, which typically contained crystals larger than $1 \mathrm{um}$. However, FTIR generally failed to locate crystalline material within finer-grained particles, especially those from the upper portions (including bulbs) of tracks.

For Synchrotron X-ray Powder Diffraction (SXRF) each particle was mounted on a thin glass fiber with a $3 \mu \mathrm{m}$ thickness using a small amount of acetone-soluble bond, set in a Gandolfi camera, and exposed to synchrotron X-rays with a wavelength of $2.161 \pm 0.001 \AA$ for 3 hours to produce a powder X-ray diffraction pattern. The analysis was performed at beam line $3 \mathrm{~A}$ of the Photon Factory, Institute of Material Science, High Energy Accelerator Research Organization and at beam line 37XU of the Japan Synchrotron Radiation Research Institute (SPring 8).

The TEM results reported have been obtained at many institutions. In Lille we used a Philips CM30 (LaB6 filament, working at $300 \mathrm{keV}$ ) and a Tecnai G2-20 twin (LaB6 filament, $200 \mathrm{kV}$ ). Structural (diffraction) data were obtained using the Selected Area Electron Diffraction (SAED) technique. Chemical compositions were measured using Energy Dispersion X-ray Spectroscopy (EDX) Noran and EDX Si-detectors (CM30 and Tecnai, respectively). Correction procedures have been applied (k-factors and absorption corrections). At Michigan State University we used a JEOL 6400 Scanning Electron Microscope (SEM) operated at 25kV, with a Noran EDX system. TEM work here was performed using a JEOL 2200FS Field Emission Gun (FEG) TEM at 200kV, with an Oxford EDX system. At Kobe University grains were studied using a TEM (JEOL JEM-2010) operated at $200 \mathrm{kV}$ and equipped with an EDX detector For quantitative TEM analyses, k-factors for the major elements were determined using standards of San Carlos olivine, San Carlos clinopyroxene, and K-feldspar from Koryu mine, Hokkaido, Japan. At Friedrich-Schiller-University Jena we used an energy-filtered 200 kV ZEISS LEO922 TEM with a ThermoNoran Six EDX system. At the University of Chicago, samples were examined with a JEOL JSM-5800 low voltage SEM equipped with an Oxford/Link ISIS-300 EDX system. We also used a Tecnai F30 TEM, with a point-to-point resolution of $0.2 \mathrm{~nm}$, operated at $300 \mathrm{kV}$. At the University of New Mexico all analyses were performed using a JEOL GEM2010 High Resolution TEM with point-to-point resolution of $0.19 \mathrm{~nm}$. It is equipped with a LINK ISIS EDX system for in situ element analyses with a $5 \mathrm{~nm}$ probe. The spectrometer is fitted with an ultrathin window for quantitative light element analyses. The instrument operated at a $200 \mathrm{keV}$ accelerating voltage. Additional analyses were performed using a JEOL 2010F FASTEM TEM/STEM instrument operating at $197 \mathrm{keV}$. The instrument is equipped with a GATAN GIF 2000 imaging filtering system and Oxford INCA/Isis EDS system. The CliffLorimer thin-film procedure was used for quantitative chemical determination with an error $<10 \%$ (relative). Crystallographic data were obtained by SAED. An LN2-cooled cold-finger was used to minimize sample degradation. Each sample was placed in a low-background double-tilt sample holder that was dedicated to this particular project to avoid contact with extraneous materials. All sample handling occurred inside a laminar flow bench. At the Naval Research Lab TEM analyses were performed using a JEOL 2200FS $200 \mathrm{kV}$ field-emission microscope equipped with a Noran System Six EDX and Gatan Ultrascan Charge-Coupled Device (CCD). At Tokyo University we used a Hitachi S-4500 FEG-SEM with EDX and Electron BackScattered Diffraction (EBSD). We also used a JEOL JEM2010 TEM with an EDX system. TEM measurements at NASA Johnson Space Center were obtained using a JEOL 2500SE 200kV FEG-STEM equipped with a Noran thin window EDX spectrometer, a Gatan Tridiem imaging filter for Energy-Filtered TEM imaging (EFTEM) and EELS, and a $2 \mathrm{~K}$ x $2 \mathrm{~K}$ slow scan 
CCD camera for recording images. Image acquisition and processing were carried out using Gatan Digital Micrograph software. EFTEM images were collected with acquisition times of 20$60 \mathrm{~s}$ depending on element concentrations. EELS spectra were obtained in image mode with spot sizes of 10-50 nm, a dispersion of $0.3 \mathrm{eV}$, dwell times of 10-30 s at an energy resolution of $0.9 \mathrm{eV}$ full width half maximum at the zero-loss peak. High resolution brightfield images were recorded at $500 \mathrm{~K}-1 \mathrm{M}$ X magnification and ordering was estimated using fast Fourier transforms of selected regions within the images.

\section{Results}

All of the Wild 2 particles we have examined thus far have been modified in various ways by the capture process, in which cometary "particles" punched into the silica aerogel capture medium, making various types of "tracks", and disaggregating into "grains" distributed along the tracks. All particles that may have been loose aggregates disaggregated into individual components with the larger, denser components penetrating more deeply into the aerogel, making thin tracks with terminal grains. Individual grains experienced heating effects that ranged widely from excellent preservation to melting; such behavior was expected (Barrett e al., 1993; Hörz e al., 2000; Burchell et al., 2006). It appears that the material deposited along track walls experienced the greatest heating, while the denser grains outran the heated areas as they lunched deeper into the aerogel (Kyoko Okudaira, personal communication 2007).

During sample PET we harvested Wild 2 grains from 52 tracks, and have obtained a rudimentary understanding of the mineralogy of 26 of these (Zolensky et al., 2006). Of the best studied 26 tracks, 8 are dominated by olivine grains (Tracks 1, 22, 26, 43, 57, 68, 71, 77), 7 by low-calcium pyroxene (Tracks 17, 20, 24, 27, 32, 41, 69), 3 by a fairly equal amount of olivine and pyroxene (Tracks $5,10,35$ ), and the remaining 8 are dominated by other minerals, mainly Fe-Ni sulfides. These results suggest that crystalline materials are abundant in Wild 2.

The recovered Wild 2 samples are mixtures of crystalline and amorphous materials. Analytical Electron Microscopy (AEM) analysis of grains from the upper, often bulb-shaped, portions of tracks show that they typically have widely varying compositions, but frequently similar to chondrites for most elements except $\mathrm{Si}$, even in severely heated and melted regions (Zolensky et al., 2006; Flynn et al., 2006; Leroux et al., submitted). The crystalline grains observed among the upper portions of individual tracks are almost always sub-micrometer in grain size. These observations suggest that the materials captured in the upper portions of the tracks are, in general, much finer grained than the material at the end of the slender, so-called stylus tracks that almost always project from the bulb-like upper tracks. AEM of very small craters on the aluminum foil also reveals crystalline olivine, pyroxene and sulfides derived from separate submicrometer components within micrometer-size particles. Synchrotron X-Ray Fluorescence (SXRF) analyses (Flynn et al., 2006), suggest that 40-100\% of the collected grains' mass is found in the upper portions of the tracks, and up to $60 \%$ is represented by the track terminal grains (on average).

- Thus our current model for the structure of the captured grains is one of very finegrained (sub-micrometer), loosely-bound aggregates with a bulk chondritic composition, with most aggregates also containing one or more much larger individual crystals (most commonly) of olivine, pyroxene and Fe-Ni sulfides.

Out of the $\sim 70$ tracks we have carefully photodocumented, only 2 appear to have no visible terminal grains. This finding indicates that practically all collected cometary particles contained some of these larger grains, which probably served to nucleate the cometary particles 
at the earliest stage of accretion. This view is supported by some of the larger crater morphologies observed on the Stardust Al foils, which have a multilobe appearance rather than being simple hemispherical craters, and which can contain diverse sub-grain compositions (Zolensky et al, 2006). This physical structure is consistent with several chondritic materials, including chondritic interplanetary dust particles (Rietmeijer, 1998; 2002).

\section{Wild 2 Olivine and Low-Calcium Pyroxene}

Olivine and pyroxene are thus present in the majority of Wild 2 particles, with observed grain size ranging from submicrometer to over $10 \mu \mathrm{m}$ (Fig. 1\&2). Wild 2 olivine has an extremely wide composition range, from $\mathrm{Fo}_{4-100}$ with a pronounced frequency peak at Fo99 (Fig 3). The presence of significant Fe-rich olivine and lack of a peak at equilibrated olivine compositions $\left(\sim \mathrm{FO}_{40-60}\right)$ suggests that composition changes due to capture heating have been insignificant in the coarse-grained $(>0.2 \mu \mathrm{m})$ component en masse (Akai, 1988; Zolensky and Kinard, 1993; Barrett et al., 1993; Hörz e al., 2000; Burchell et al., 2006). Wild 2 olivines include some with very elevated $\mathrm{MnO}, \mathrm{Al}_{2} \mathrm{O}_{3}$ and $\mathrm{Cr}_{2} \mathrm{O}_{3}$ contents, up to $6.45,0.71$ and $1.46 \mathrm{wt} \%$, respectively (Zolensky et al., 2006). About $1 / 4$ of these $\mathrm{Mn}$ - and Cr-rich olivines contain $<<1 \%$ $\mathrm{FeO}$. Olivines with enrichments in these elements have been reported in carbonaceous chondrites, micrometeorites, and chondritic IDPs, though they are rare (Klöck et al., 1989; Rietmeijer, 1998; Gounelle et al., 2002; Simon and Grossman, 2003; Weisberg et al., 2004). The wide $\mathrm{Mg}-\mathrm{Fe}$ composition range of Wild 2 olivine is similar to that for anhydrous chondritic IDPs. However, the range of these olivine compositions is also similar to what is found in the matrix of the chondrites Murchison (CM2), and Orgueil (CI1), which have experienced significant-topervasive aqueous alteration. The presence of the pronounced peak in forsterite seen in the CMs could be due to the preferential survival of Mg-rich olivine during aqueous alteration, a wellknown phenomenon since Fe-rich olivine is significantly more susceptible to dissolution, and alteration than Mg-rich olivine (Wogelius and Walther, 1992). The olivine analyses alone, however, do not demonstrate whether Comet Wild 2 ever hosted liquid water. Particular Wild 2 particles which have the most Mg-rich olivine, and the most restricted olivine compositions are potentially survivors of localized, partial aqueous alteration. The resemblance of Wild 2 olivine to $\mathrm{CI}$ and $\mathrm{CM}$ chondrite matrix olivine with pronounced forsterite peaks, suggests that a more detailed search for possible aqueous alteration products should be undertaken.

Both low- and high-calcium pyroxenes are present among the Wild 2 grains, with the former being dominant. In this paper we will deal only with the volumetrically dominant lowcalcium pyroxenes. In some cases Synchrotron X-ray Diffraction (SXRD) or Selected Area Electron Diffraction (SAED) patterns reveal low-calcium pyroxenes to be orthoenstatite, requiring slow cooling, but in the majority of cases we have only EDX analyses and are not certain whether we have ortho- or clinopyroxene. The composition range displayed by the lowcalcium pyroxene is also very extensive, from $\mathrm{En}_{52}$ to $\mathrm{En}_{100}$, with a significant frequency peak centered at $\mathrm{En}_{95}$ (Fig. 4). Low-calcium pyroxene usually coexists with olivine in the Wild 2 grains, but the $\mathrm{Mg} / \mathrm{Fe}$ ratios for coexisting phases are not always similar. For example, Track 17 contains olivine in the range $\mathrm{Fo}_{55-69}$, while associated low-calcium pyroxene is $\mathrm{En}_{52-96}$. Flash heating during sample collection may account for the disparity in this particular instance, as olivine equilibrates faster than orthopyroxene under identical circumstances (Ganguly and Tazzoli, 1994). We have seen composition equilibration of ferromagnesian minerals in chondritic IDPs during capture in silica aerogel in low-Earth orbit aboard the Mir space station 
(Hörz et al., 2000). In this situation we observed that olivines and pyroxenes sometimes equilibrated at compositions in the range $\left(\sim \mathrm{Fo}_{40}\right.$ to $\left.\mathrm{Fo}_{60}\right)$, and sometimes recrystallize into a granular texture. This homogenization/ recrystallization also occurs within most micrometeorites during atmospheric entry (Klöck and Stadermann, 1994). The extreme composition range of low-Ca pyroxene is again similar to the anhydrous chondritic IDPs, and significantly broader than what is observed for most chondrites, including Murchison and Orgueil (Fig. 4).

It is of course important to determine the nature of any amorphous cometary silicates that may be present in the Wild 2 samples. Although amorphous silicates are common in the aerogel, thus far it has not been possible to satisfactorily establish which, if any, of this material is indigenous to the comet, a point well addressed by Leroux et al. (submitted a, b) and Chi et al. (2007). It also is possible that some of the crystalline silicates in Wild 2 are have been annealed from earlier poorly crystalline solids, a critical issue for modelers of the early solar system (Wooden et al.), but this is a question beyond the scope of the present paper.

\section{Fe-Ni Sulfides in Wild 2 Grains}

Sulfides are the only mineral group found in all extraterrestrial materials. Fe-Ni sulfides are also ubiquitous in the Wild 2 grains, grading from sulfides which apparently melted during collection and separated into a mixture of sulfide and metal (Fig. 5\&6) (Leroux et al., submitted a, b; Rietmeijer et al, submitted), all the way to apparently unmodified FeS and pentlandite $\left((\mathrm{Fe}, \mathrm{Ni})_{9} \mathrm{~S}_{8}\right)$ grains. Several tracks (e.g. Track 59) have FeS- or pentlandite-dominated terminal grains. In this paper we collectively refer to troilite (stoichiometric $\mathrm{FeS}$ ) and pyrrhotite $\left(\mathrm{Fe}_{1-\mathrm{x}} \mathrm{S}\right.$ ) as "FeS" because the exact stoichiometry and structure is unknown in most instances. A plot of analyses of Wild 2 Fe-Ni sulfides (Fig. 7) shows that many have compositions close to that of $\mathrm{FeS}$, with less than 2 atom \% Ni. To date, only two pentlandite grains have been found among the Wild-grains suggesting that this mineral is not abundant. The complete lack of compositions between FeS and pentlandite (with intermediate solid solution compositions) suggests (but does not require) that $\mathrm{FeS}$ and pentlandite condensed as crystalline species, i.e. did not form as amorphous phases, which later became annealed (Vaughan and Craig, 1978). (A rare sulfide IDP ( $\sim 10$ microns) that is an intimate coarse-grained intergrowth of pyrrhotite and pentlandite (Rietmeijer, 2004) shows pyrrhotite and pentlandite coexist as an original assemblage in extraterrestrial material without the intermediary compositions.) The remaining Wild 2 Fe-Ni-S bearing phases (approximately half analyzed so far) have compositions that trend from FeS directly towards the Fe apex of the Fe-Ni-S ternary diagram (Fig. 7). SAED patterns for these phases show the presence of two different lattices- strong maxima for a Fe-Ni sulfide phase and a much finer pattern consistent with kamacite (Leroux et al, submitted a, b). These phases are almost certainly a result of capture heating, and their presence could be used to gauge the degree of capture modification of the enclosing Wild 2 grains.

The few verified pentlandite crystals in Wild 2 tracks are intriguing since this phase is frequently an indicator of low-temperature metamorphism under oxidizing conditions, and/or aqueous alteration (Zolensky and Thomas, 1995; Bullock et al., 2005). So far we have not observed tochilinite, which is a hallmark of the hydrated CM2s and a few hydrous chondritic IDPs (Bradley and Brownlee, 1991; Zolensky et al. 1993). Ni-rich sulfides are not found in totally anhydrous chondritic IDPs, but are found in IDPs that experienced aqueous alteration, and are abundant in carbonaceous chondrites that are aqueously altered. Therefore, at face value the presence of these few Ni-rich sulfides suggests that some Wild 2 particles could have experienced a limited degree of aqueous alteration. 


\section{Caveats}

In the course of making comparisons to chondrite matrix and IDP silicates, we have been reminded that there are still very few good olivine and pyroxene analyses from the Wild 2 samples, especially as regards minor elements. Also, most of the few available analyses of chondrite matrix olivine or pyroxene derive from microprobe analyses of matrix which is generally too fine-grained to support such analyses. Of course some good olivine and pyroxene analyses have been obtained from coarse-grained matrix material, but in general the old probe analyses are simply not useful here. For some major meteorite types matrix analyses are not even available. The problem is probably most acute for the ordinary-, enstatite-, and R chondrites. We need chondrite matrix mineral analyses based upon TEM analyses. How about our assumption that chondrite matrix is the material to which we should be making comparisons rather than microchondrules, or amoeboid olivine aggregates, or fine-grained chondrule rims? Comparisons to micrometeorites should be made, but the majority of these are highly heated and equilibrated by atmospheric entry. Even the comparison to the IDPs is to some degree questionable. Although the most extensive database of olivine and pyroxene analyses (Zolensky and Barrett, 1994) indicates that anhydrous chondritic IDPs have no significant composition peak at forsterite, bulk analyses of anhydrous chondritic IDPs indicate a high $\mathrm{Mg} / \mathrm{Fe}$ ratio (Bradley, 1988; Lawler et al., 1989). Obviously new analyses of olivine and pyroxene IDPs are also required. The dearth of minor element analyses of matrix, micrometeorite and IDP silicates is also a problem. A hallmark of the sudden availability of a new sample type is that critical discrepancies in our understanding of existing samples become glaringly apparent. It is clear that much work remains to be done on both the Wild 2 grains as well as the other fine-grained astromaterials in our collections.

\section{Expected survival of phyllosilicates during capture heating in aerogel}

To be perfectly clear, no direct evidence of phyllosilicates has yet been seen in any Wild 2 samples (Zolensky et al., 2006). Despite the fact that significant heating and structural modification accompanied collection of many grains in the aerogel, we should have seen characteristic compositions, grain morphologies, and lattice fringes of phyllosilicates or carbonates had they been present (Barrett et al., 1992, 1993; Hörz et al., 2000). As a critical aspect of the groundwork for the Stardust Mission, we performed simulations of comet dust capture experiments into silica aerogel using JSC's light gas gun in the early 1990s (Barrett et al., 1992). In these shots we used aerogel with a density of $40 \mathrm{mg} / \mathrm{cc}$, which is denser than the material we used on the Stardust Mission (30 mg/cc and lower), and we made shots up to 7.2 $\mathrm{km} / \mathrm{s}$, which was well in excess of the capture velocity at Wild $2(6.2 \mathrm{~km} / \mathrm{s})$. Despite these more destructive simulations, phyllosilicate textures and even fully hydrated phyllosilicate crystallites survived in all of our capture experiments. Figure 8 shows a typical olivine grain we fired, before and after capture in the aerogel. It happened that these olivine grains contained thin regions of serpentine and saponite within fractures and dislocations, which is commonly the case for terrestrial olivine. Despite the changes wrought to the captured grain that are obvious by inspection of Figure 8, it retained the phyllosilicates it carried, as shown by the TEM images in Figure 9. In some cases the phyllosilicates were dehydrated during capture, but here the distinctive, flaky morphology of the phyllosilicates was preserved. In other cases, the phyllosilicates retained their interlayer $\mathrm{OH}$, as evidenced by the undisturbed layer lattice spacings (Fig. 9). We also observed materials intermediate to these two survival end-members. 
In all cases we could unequivocally conclude that phyllosilicates were originally present in the captured grains. We expect to see the same effect in the Wild 2 grains, if phyllosilicates were present in the pre-capture particles.

We have observed the same phenomenon in naturally-heated (metamorphosed) carbonaceous chondrites. We have for many years been making a special investigation of the changes wrought to phyllosilicates, carbonates and $\mathrm{Fe}-\mathrm{Ni}$ sulfides during heating (metamorphism) on asteroids (Tonui et al., submitted), and the impact of a CM chondrite meteorite onto the Moon, which was brought to Earth by the Apollo 12 astronauts (Zolensky, 1997). The mineralogical changes that occurred during shock and heating of phyllosilicates in carbonaceous chondrites were first noted in a series of papers by J. Akai (Akai, 1988, 1990), were then observed by many other investigators, and have been most recently summarized by Tonui et al. (submitted). There are now more than a dozen known naturally metamorphosed carbonaceous chondrites, and the progression of mineralogical changes attending heating are fairly well understood. Here we see the same situation that we observed in the lab shots described above. During heating, phyllosilicates lose interlayer water and $\mathrm{OH}$, and interlayer lattice fringes collapse in a characteristic manner. Eventually, a neoformed anhydrous silicate is formed, generally orthopyroxene or olivine. The neoformed silicates retain the flaky morphologies of the original phyllosilicates, however, and so are still recognizable as transformed phyllosilicates.

Okudaira et al. (2004, 2006) launched phyllosilicates (serpentine and cronstedtite) into aerogel at velocities of 4 and $6.1 \mathrm{~km} \mathrm{sec}^{-1}$, and saw preservation of a significant fraction. Additional evidence that some phyllosilicates would have survived capture is presented by the partial survival of scattered, delicate, low temperature phases among the Wild 2 samples - finegrained, organic rich phases rich in deuterium and ${ }^{15} \mathrm{~N}$ excesses (Sandford et al, 2006; Cody et al., 2007). These fine-grained materials survived capture in the aerogel with minimal heating when attached to larger, more refractory grains. Phyllosilicates would have behaved similarly.

In these carbonaceous chondrites carbonates devolatilize during metamorphism to hydroxides or oxides (Tonui et al., 2002). We have also observed the devolatilization products of carbonates within hydrous IDPs that have been seriously heated during atmospheric entry (Zolensky et al, 1992). Ca-Mg-Fe carbonates in these IDPs boil down to Ca-Mg-Fe hydroxides and finally oxides. As indicated above, we have seen the same process occurring to chondritic IDPs captured in silica aerogel in low-Earth orbit aboard the Mir space station (Hörz et al., 2000), and within most micrometeorites during atmospheric entry (Klöck and Stadermann, 1994).

So, serpentine, saponite and Ca carbonates have been successfully captured in silica aerogel even at velocities $1 \mathrm{~km} / \mathrm{s}$ higher than those experienced at Wild 2, in both laboratory simulations and actual IDP collection in Earth orbit aboard the MIR space station (Barrett et al., 1992, 1993; Hörz et al., 2000). In instances where phyllosilicates have been dehydrated, rendered amorphous, or recrystallized during capture in silica aerogel, characteristic grain morphologies and basal lattice spacings are formed which signal the original mineralogy. Thus the lack of phyllosilicates, and rarity of carbonates (Wirick et al., 2007; Mikouchi et al., 2007) among the $\sim 50$ Wild 2 grains we have so far well characterized suggests that they could not have composed more than a few percent of the more coarse-grained fraction of captured Wild 2 sample. However, it is also true that for the most part only the most coarse-grained objects at the end of capture tracks have been analyzed to a significant degree during Stardust sample preliminary examination (PET), and that these have for the most part been incompletely characterized (only "exciting" grains like the CAI particle have received detailed analysis so far). 
It is also likely that many phyllosilicate-rich (and other $\mathrm{OH}$-bearing phases) grains, if ever present among captured Wild 2 particles, would have been deposited along the walls of bulb tracks as well as at track termini. These wall grains have been little studied during PET. Therefore more thorough characterization of bulb wall grains as well as the terminal grains will be necessary to definitively determine whether Comet Wild 2 ever hosted liquid water. Comprehensive work on bulb wall grains was not performed adequately during sample PET because these grains were the most thoroughly broken, and in some cases most heated, during capture into the aerogel, and because they were never the largest captured grains. In addition, in the rush to do sample PET the kind of detailed TEM work needed to reveal the presence of minor phyllosilicates among large terminal grains was really not adequately performed.

To conclude, if phyllosilicates had dominated the mineralogy of the captured samples, we would probably have seen strong evidence of this already. But if aqueous alteration has affected much less than half of the comet materials we would probably have missed it during sample PET. A critical question we must address in future work is: did the comet nucleus ever support liquid water, and if so to what extent?

\section{Acknowledgements}

We thank Eric Tonui and George Flynn for thoughtful reviews which significantly improved the manuscript. All authors thank their respective funding agencies. We are particularly indebted to NASA for funding the Stardust Discovery Mission and the subsequent preliminary examination of the returned Wild 2 samples through a Participating Scientist Program and the Stardust Data Analysis Program, which supported many of us.

\section{References}

Akai J. (1988) Incompletely transformed serpentine-type phyllosilicates in the matrix of Antarctic CM chondrites. Geochim. Cosmochim. Acta 52, 1593-1599.

Akai J. (1990) Mineralogical evidence of heating events in Antarctic carbonaceous chondrites, Y-86720 and Y-82162. Proceedings of the NIPR Symposium on Antarctic Meteorites, No. 3, 55-68.

Barrett R.A., Zolensky M.E. and Bernhard R. (1993) Mineralogy of chondritic interplanetary dust particle impact residues from LDEF. Lunar and Planetary Science XXIV, The Lunar and Planetary Institute, 65-66.

Barrett R.A., Zolensky M.E., Hörz F., Lindstrom D.J., Gibson E.K., Proceedings of the 19th Lunar and Planetary Science Conference, 22, 203 (1992).

Bradley J.B. (1988) Analysis of chondritic interplanetary dust thin-sections. GCA 52, 889-900.

Bradley J.B. (1994) Chemically anomalous, preaccretionally irradiated grains in interplanetary dust particles. Science 265, 925-929.

Bradley J.B. and Brownlee D.E. (1991) An interplanetary dust particle linked directly to CM meteorites and an asteroidal origin. Science 251, 549-552.

Brearley A. (1989) Nature and origin of matrix in the unique type 3 chondrite, Kakangari. GCA 53, 2395-2411.

Brearley A.J. (1993) Matrix and fine-grained rims in the unequilibrated CO3 chondrite, ALHA77307; Origins and evidence for diverse, primitive nebular dust components. Geochim. Cosmochim. Acta 57, 1521-1550 (1993). 
Bullock E.S., Gounelle M., Lauretta D.S., Grady M.M., and Russell S. (2005) Mineralogy and texture of Fe-Ni sulfides in CI1 chondrites: Clues to the extent of aqueous alteration on the CI1 parent body. GCA 69, 2687-2700.

Burchell M.J., Graham G., Kearsley A. (2006) Cosmic dust collection in aerogel. Annual Review of Earth and Planetary Sciences 34, 385.

Chi M., Ishii H., Dai Z.R.1, Toppani A., Joswiak D.J., Leroux H., Zolensky M., Keller L.P., Browning N.D., and Bradley J.P. (2007) Does Comet Wild 2 contain GEMS? Lunar and Planetary Science XXXVIII, abstract 2010.

Cody G.D., Yabuta1 H.,. Alexander C.M.O’d, Araki T., Kilcoyne A.L.D., and The Stardust Pet TEAM (2007) Placing Comet 81p/Wild 2 Organic Particles Into Context With Chondritic Organic Solids. Lunar And Planetary Science XXXVIII, Abstract 2286.

Flynn G. et al. (2006) Elemental Compositions of Comet 81P/Wild 2 Samples Collected by Stardust, Science 314, 1730-1733..

Fuchs L., Olsen E. and Jensen K. (1973) Mineralogy, mineral-chemistry and composition of the Murchison (C2) meteorite. Smithsonian Contribs. Earth Sci. 10, 39p.

Ganguly M., Tazzoli V. (1994) $\mathrm{Fe}^{2+}-\mathrm{Mg}$ interdiffusion in orthopyroxene: retrieval from the data on intracrystalline exchange reaction American Mineralogist 79, 930 (1994).

Gounelle M., Devouard B., Engrand C., Genge M. , Topani A., Leroux, H. Meteoritics Planetary Science 37, A55 (2002).

Gounelle M., Spurny P., and Bland P. A. (2006) The atmospheric trajectory and orbit of the Orgueil meteorite. Meteoritics Planet. Sci. 41, 135-150.

Greshake A (1997) The primitive matrix components of the unique carbonaceous chondrite Acfer 094: a TEM study. Geochim. Cosmochim. Acta 61, 437-452.

Hörz F., Zolensky M.E., Bernhard R.P., See T.H., and Warren J.L. (2000) Impact features and projectile residues in aerogel exposed on Mir. Icarus 147, 559-579 (2000).

Ishii H.A., Graham G.A., Kearsley A.T., Grant P.G., Snead C.J., Bradley J.P. (2005) MAPS 40, 1741(2005).

Kerridge J. and MacDougal J. (1976) Mafic silicates in the Orgueil carbonaceous meteorite. EPSL 29, 341-348.

Klöck W., Thomas K.L., McKay D.S., Palme P. (1989) Unusual olivine and pyroxene compositions in interplanetary dust and unequilibrated ordinary chondrites. Nature 339, 126-128.

Klöck W. and Stadermann F.J. (1994) Mineralogical and chemical relationships of interplanetary dust particles, micrometeorites and meteorites. In Analysis of Interplanetary Dust. M.E. Zolensky, T.L. Wilson, F.J.M. Rietmeijer and G.J. Flynn, Eds., AIP Press, pp. 51-87.

Krot A.N., Scott E.R.D. and Zolensky M.E. (1995) Mineralogic and chemical variations among CV3 chondrites and their components: Nebular and asteroidal processing. Meteoritics 30, 748-775.

Lawler M.E, Brownlee D.E., Temple S., and Wheelock M.M. (1989) Iron, magnesium, and silicon in dust from Comet Halley. Icarus 80, 225.

Leroux H., Rietmeijer F. J. M, Velbel M. A., Brearley A. J., Jacob D, Langenhorst F., Bridges J. C., Zega T. J., Stroud R. M., Cordier P., Harvey R. P., Lee M., Gounelle M., and Zolensky M.E. A TEM study of thermally modified Comet $81 \mathrm{P} /$ Wild 2 dust particles by interactions with the aerogel matrix during the Stardust capture process. Meteoritics and Planetary Science, Submitted a. 
Leroux H., Stroud R. M., Dai Z. R., Graham G., Troadec D., Bradley J., Teslich N., Borg J., Kearsley A. and Hörz F. 2007b. Transmission electron microscopy of cometary residues from micron-sized craters in the Stardust Al-foils. Meteoritics and Planetary Science, Submitted b.

Lisse C., VanCleve J., Adams A., Ahearn M., Fernández Y., Farnham T, Armus L., Grillmair. C, Ingalls J., Belton M.., Groussin O., McFadden L., Meech K., Schultz P., Clark B., Feaga L., Sunshine J. (2006) Spitzer Spectral Observations of the Deep Impact Ejecta. Science 313, 635.

Lisse, C.M., Bodewits, D., Christian, D.J., Wolk, S.J., Dennerl, K., Zurbuchen, T.H., Hansen, K.C., Hoekstra, R., Combi, M., Fry, C.D., Dryer, M., Makinen, T., and Sun, W. (2007) Chandra observations of Comet 9P/Tempel 1 during the Deep Impact campaign. Icarus 187, 69-84.

Matsunami S. et al. (1990) The chemical compositions and textures of matrices and chondrule rims of unequilibrated chondrites - II. Their constituents and implications for the formation of matrix olivine. Proc NIPR Symp. Antarct. Mets. 3, 147-180;

Mikouchi T., Tachikawa O., Hagiya K., Ohsumi K, Zolensky M. (2007) Mineralogy and crystallography of Comet $81 \mathrm{P} /$ Wild 2 particles returned by the Stardust Mission. Lunar And Planetary Science XXXVIII.

Nagahara H. (1984) Matrices of type 3 ordinary chondrites - primitive nebular records. GCA 48, 2581-2595

Nier A.O. and Schlutter D.J. (1990) Helium and neon isotopes in stratospheric particles. Meteoritics 25, 263-267.

Nier A.O. and Schlutter D.J. (1992) Extraction of helium from individual interplanetary dust particles by step-heating. Meteoritics 27, 166-173.

Nier A.O. and Schlutter D.J. (1993) Thye thermal history of interplanetary dust particles in the Earth's stratosphere. Meteoritics 28, 675-681.

Okudaira K., Noguchi T., Nakamura T., Sugita S., Sekine Y. and Yano H. (2004) Evaluation of mineralogical alteration of micrometeoroid analog materials captured in aerogel. Advances in Space Research 34, 2299-2304.

Okudaira K., Yano H., Noguchi T., Nakamura T, Burchell M.J. And M. J. Cole M.J. (2006) Are They Really Intact? --- Evaluation Of Captured Micrometeoroid Analogs by Aerogel At the Flyby Speed Of Stardust. Lunar And Planetary Science XXXVII, Abstract 1832.

Reid, A.M., Bass, M.N. Fujita, H., Kerridge, J.F. and Fredriksson, K.. (1970) Olivine and pyroxene in the Orgueil meteorite. GCA 34, 1253-1255.

Rietmeijer F.J.M. (1998) Interplanetary Dust Particles. In Planetary Materials, Reviews in Mineralogy, vol. 36 (J.J. Papike, ed.), 2-1 - 2-95. Mineralogical Society of America, Chantilly, Virginia.

Rietmeijer F.J.M. (2002) The earliest chemical dust evolution in the solar nebula. Chemie der Erde 62, 1-45.

Rietmeijer F.J.M. (2004) Dynamic pyrometamorphism during atmospheric entry of large $(\sim 10$ micron) pyrrhotite fragments from cluster IDPs. Meteoritics and Planetary Science 39, 1869-1887.

Sandford S.A., Aléon J., Alexander C.M.O’D., Araki T., Bajt S., Baratta G.A., Borg J., Brucato J.R., Burchell M.J., Busemann H., Butterworth A., Clemett S.J., Cody G., Colangeli L., Cooper C., D'Hendecourt L., Djouadi Z., Dworkin J.P., Ferrini G., Fleckenstein H., Flynn G.J., Franchi I.A., Fries M., Gilles M.K., Glavin D.P., Gounelle M., Grossemy F., 
Jacobsen C., Keller L.P., Kilcoyne A.L., Leitner J., Matrajt G., Meibom A., Mennella V., Mostefaoui S., Nittler L.R., Palumbo M.E., Robert F., Rotundi A., Snead C.J., Spencer M.K., Steele A., Stephan T., Tyliszczak T., Westphal A.J., Wirick S., Wopenka B., Yabuta H., Zare R.N., and Zolensky M. (2006) Organics Captured from Comet Wild 2 by the Stardust Spacecraft. Science 314, 1720-1724.

Simon S.B and. Grossman L. (2003) Petrography and mineral chemistry of the Tagish Lake carbonaceous chondrite. Meteoritics Planetary Science 38, 813-825.

Tonui E., Zolensky M., Hiroi T., Nakamura T., Lipschutz M.E., Wang M.-S.and Okudaira K. Petrographic, chemical and spectroscopic evidence for thermal metamorphism in carbonaceous chondrites 1: CI and CM chondrites. Submitted to GCA.

Tonui E., Zolensky M.E. and Lipschutz M.E. (2002) Petrography, mineralogy and trace element chemistry of Y-86029, Y-793321 and LEW 85332: Aqueous alteration and heating events. Proceedings of the NIPR Symposium on Antarctic Meteorites, No. 15, 38-58.

Vaughan D., Craig J., Mineral Chemistry of Metal Sulfides. Cambridge Univ. Press., 493 (1978).

Weisberg M.K., Connolly H.C., Ebel D.S. (2004) Petrology and Origin of Amoeboid Olivine Aggregates in CR Chondrites. Meteoritics and Planetary Science, 39: 1741-1753.

Westphal A.J. et al. (2004) Meteoritics \& Planetary Science 39, 1375.

Wirick S., Leroux H., Tomeoka K., Zolensky M, Flynn G., Tyliszczak T., Butterworth A, Tomioka N., Ohnishi I, Nakamura-Messenger K, Sandford S, Keller L., Jacobsen C. (2007) Carbonates Found in Stardust Aerogel Tracks. Lunar And Planetary Science XXXVIII.

Wogelius R.A. and Walther J.V., (1992) Olivine dissolution kinetics at near surface conditions: Chem.Geol. 97, 101-112.

Wooden et al. Chondrules and the Protoplanetary Disk.

Zolensky M.E. (1997) Structural water in the Bench Crater chondrite returned from the Moon. Meteoritics and Planetary Science 32, 15-18.

Zolensky M.E. and Barrett R.A. (1994) Composition variations of olivines and pyroxenes in chondritic interplanetary dust particles. Meteoritics 29, 616-620 (1994).

Zolensky M.E., Barrett R.A. and Browning L. (1993) Mineralogy and composition of matrix and chondrule rims in carbonaceous chondrites. Geochimica et Cosmochimica Acta 57, 31233148.

Zolensky M.E. and Kinard W.H. (1993) Results of the LDEF meteoroid and debris special investigation group. Advances in Space Research 13, (8)75-(8)85.

Zolensky M.E. and Lindstrom D.J. (1992) Mineralogy of 12 large "chondritic" interplanetary dust particles. Proceedings of the 19th Lunar and Planetary Science Conference, G. Ryder and V. Sharpton, Eds., 161-169.

Zolensky M.E., Prinz M. and Lipschutz M.E. (1991) Mineralogy and thermal history of Y-86720, Y-86720 and B-7904. 16th Symposium on Antarctic Meteorites, 78-2.

Zolensky M.E. and Thomas K. (1995), Iron and iron-nickel sulfides in chondritic interplanetary dust particles. Geochim. Cosmochim. Acta 59, 4707-4712.

Zolensky M. et al. (2006) Mineralogy and Petrology of Comet Wild 2 Nucleus Samples. Science 314, 1735-1740. 


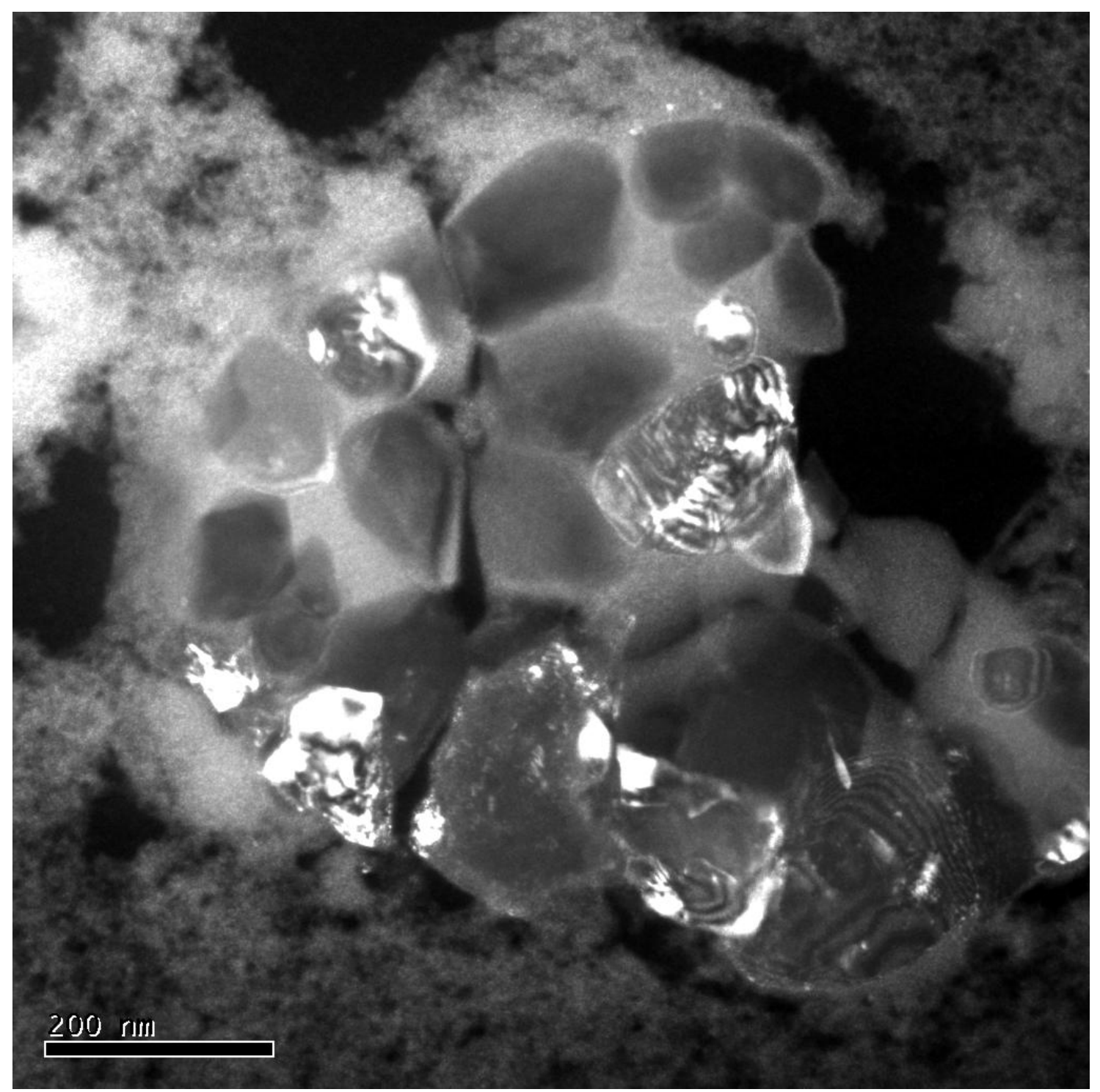

Figure 1. Bright field TEM image showing an olivine and pyroxene sample. Individual olivine and pyroxene grains crystallites are srrounded by amorphous matrix (which may or may have been produced during capture). 


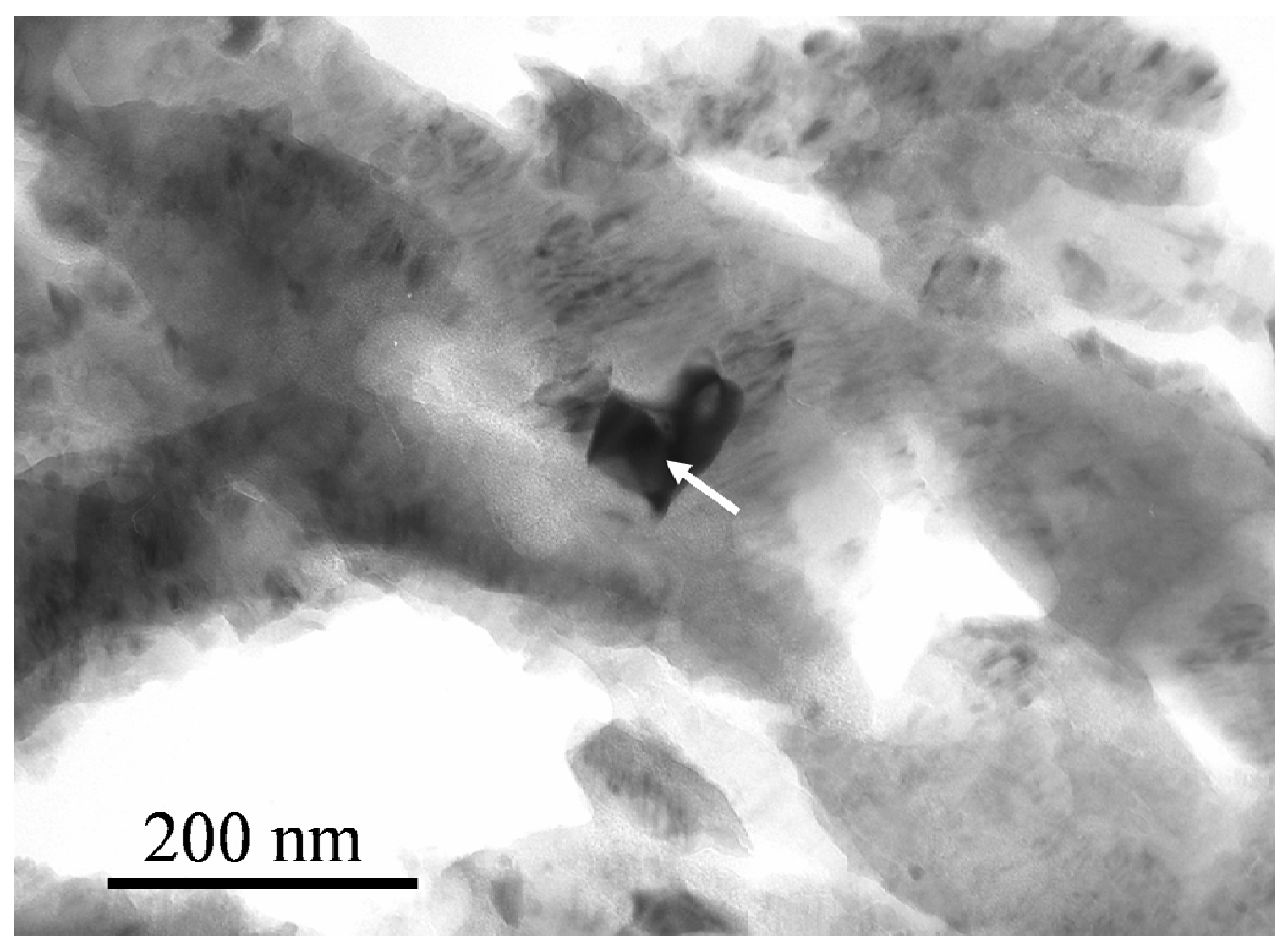

Figure 2. Bright field TEM image showing a pyroxene-rich sample $\left(\mathrm{En}_{77} \mathrm{Wo}_{4} \mathrm{Fs}_{19}\right)$ containing minor and small olivine ( $\mathrm{Fo}_{77}$ ) grains (arrowed). Sample C2027,2,69,1,1. 

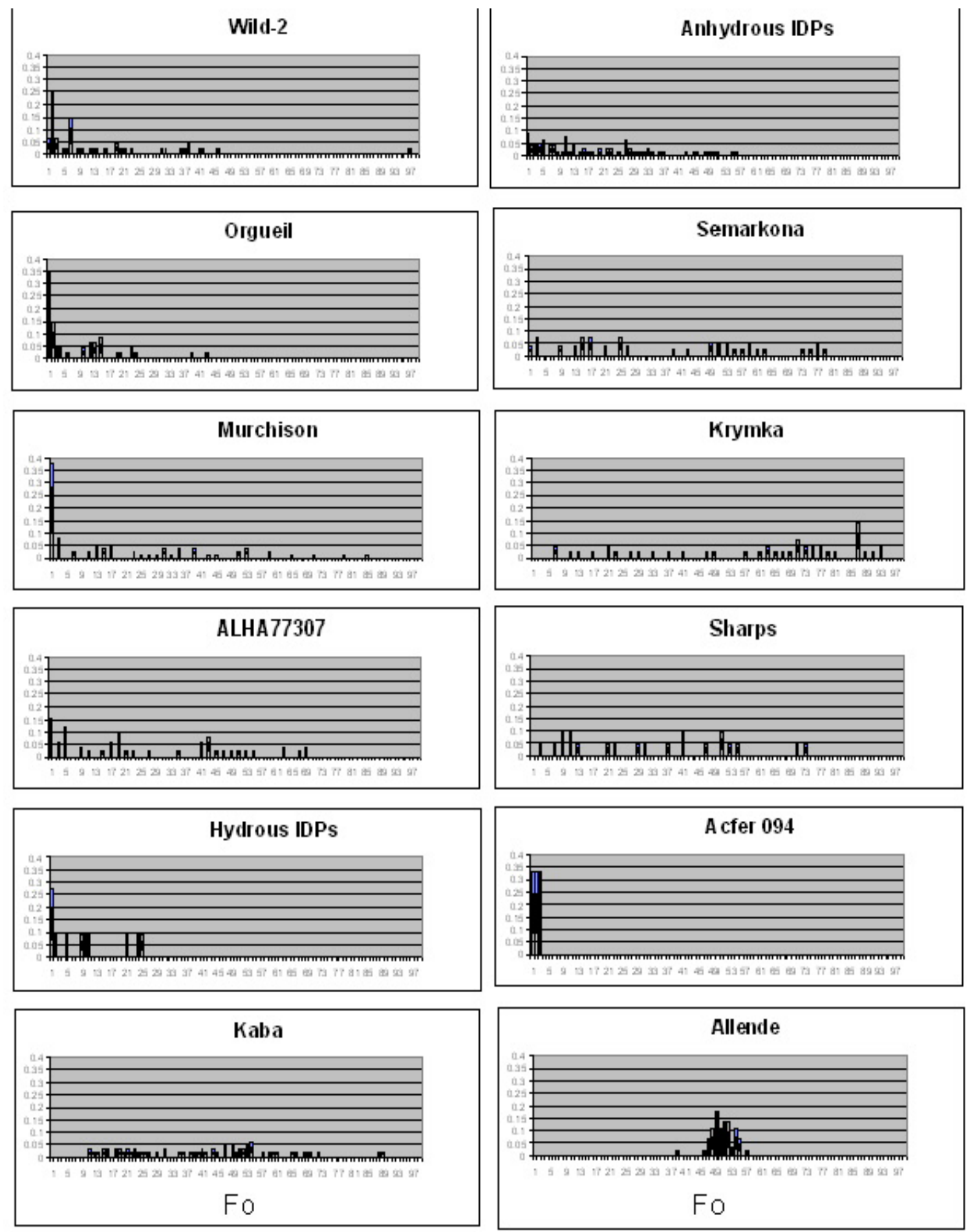

Figure 3. Composition ranges of olivine (Fo) in grains from 9 Wild 2 particles, compared with those for chondritic IDPs (Zolensky and Barrett, 1994) and primitive chondrite matrix olivine (Greshake, 1997; Brearley, 1993; Reid et al., 1970; Kerridge J. and MacDougal J. (1976); Krot et al., 1995; Fuchs et al., 1973; Nagahara, 1984; Matsunami et al., 1990). Vertical scale is number of analyses normalized to a total of 1 . 

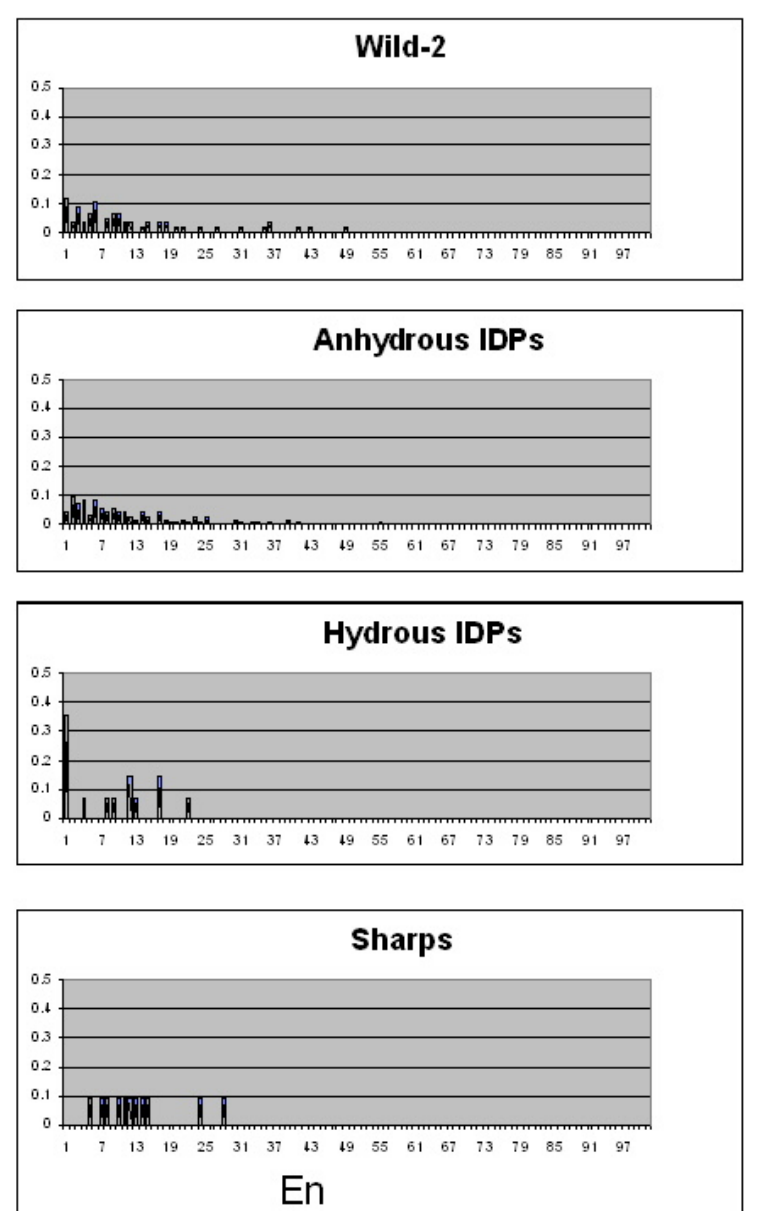
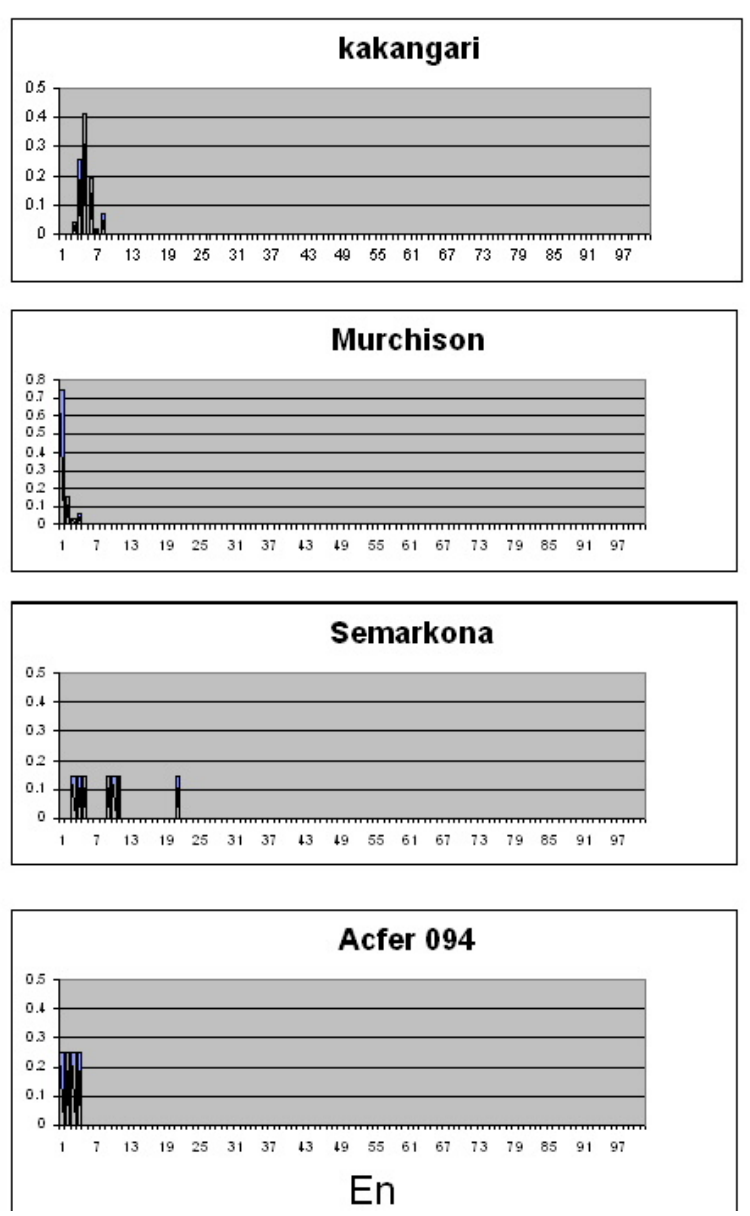

Figure 4. Composition ranges of low-Ca pyroxene (En) in grains from Wild 2 particles, compared with those for chondritic IDPs and primitive chondrite matrix pyroxene, from the same references as Fig. 1. 


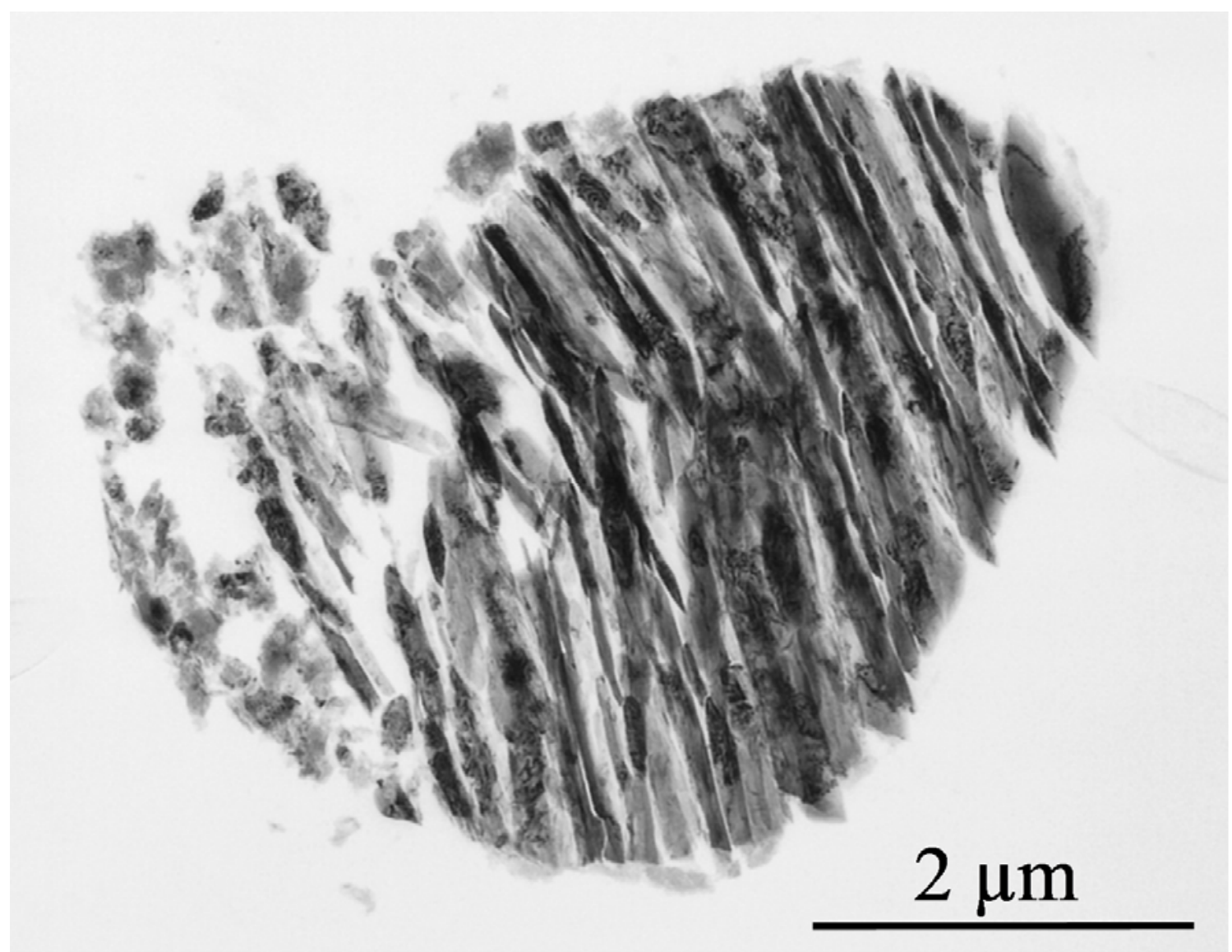

Figure 5a 


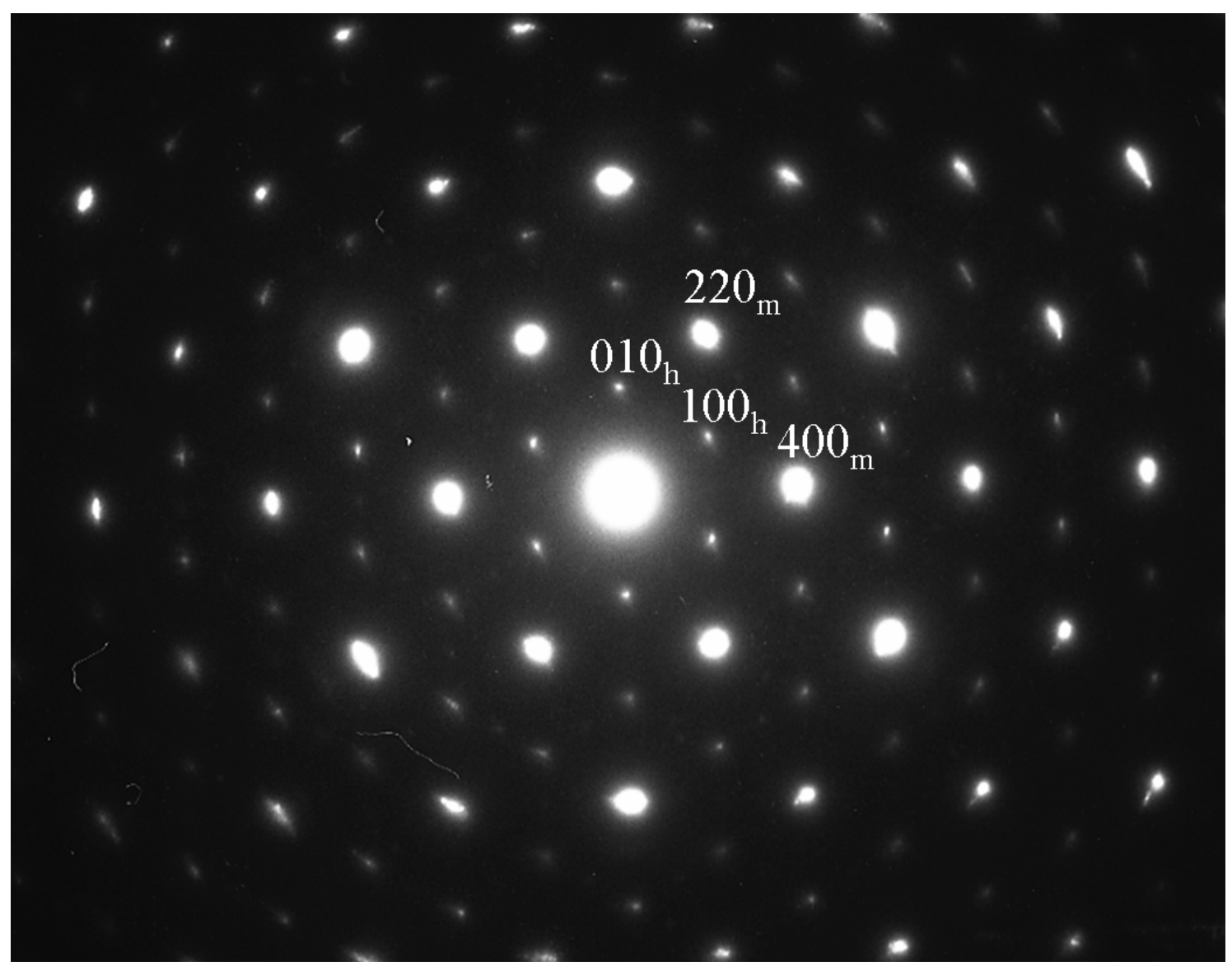

Figure $5 b$

Figure 5. Pyrrhotite grain in sample C2004,0,36,1,5 (a) TEM bright field image showing the entire grain (b) Selected area electron diffraction of the grain. The structure of pyrrhotite can be described with a hexagonal structure (slightly distorted), $\mathrm{a}=0,588$ and $\mathrm{c}=1.139 \mathrm{~nm}$, or with a monoclinic structure $a^{\prime}=1.190, b^{\prime}=0.686, c^{\prime}=2.279 \mathrm{~nm}, \beta=90.43$ (strong spots). 


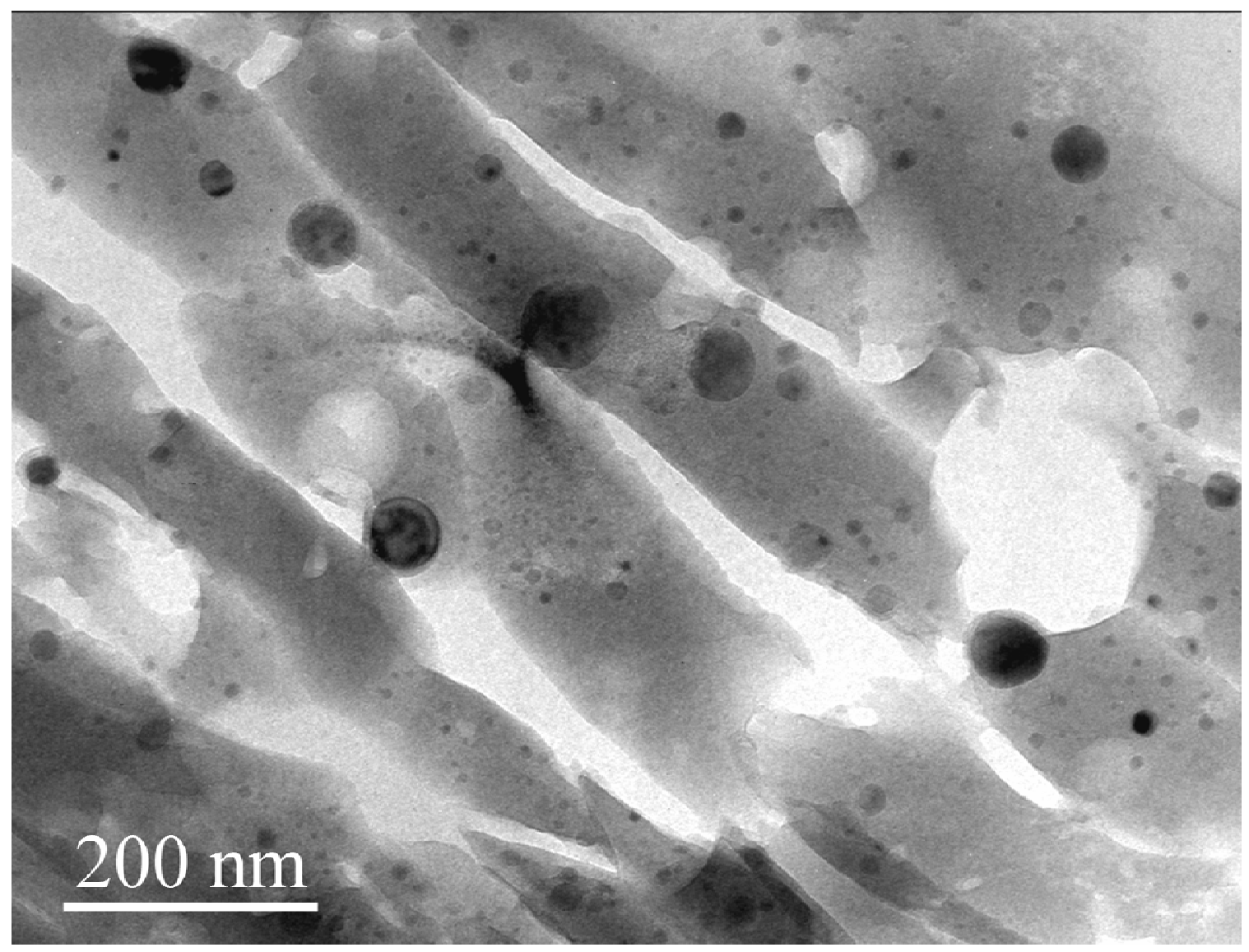

Figure 6. TEM bright field image showing a thermally modified sample (C2004,1,44,4,2) which consist of a glassy matrix with Fe-Ni-S inclusions. The chemistry of these samples is strongly dominated by silica, suggesting a mixture between molten aerogel and the incident particle. 


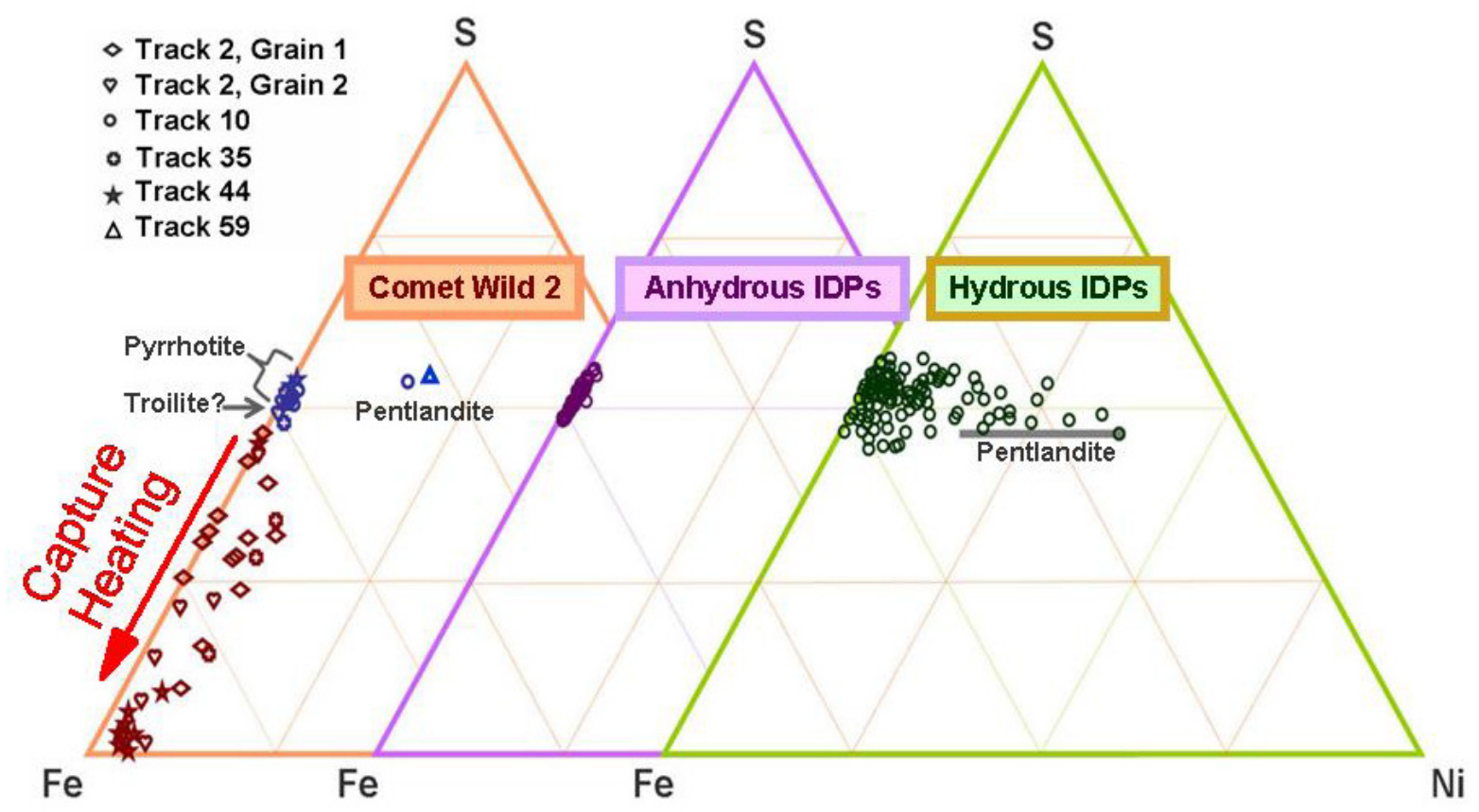

Figure 7. Composition ranges of Fe-Ni sulfides from 5 grains from 4 Wild 2 particle tracks. Grains from track walls as well as track termini were analyzed. Most Wild 2 sulfides are probably a mixture of troilite and pyrrhotite, and one grain of pentlandite is present. Many sulfides plot with nonstoichiometric, low $\mathrm{S}$ compositions reflecting capture heating. The corresponding composition ranges for hydrous and anhydrous chondritic IDPs (Zolensky and Thomas, 1995) are also shown. Anhydrous chondritic IDPs contain only troilite and pyrrhotite, while the hydrous chondritic IDPs also have equally-abundant Ni-rich sulfides including pentlandite. With the exception of the two identified pentlandite crystals, the Wild 2 grains have the same Fe-Ni sulfide composition range as the anhydrous chondritic IDPs. However, the presence of pentlandite suggests the possibility that aqueous alteration has occurred to a minor degree. 


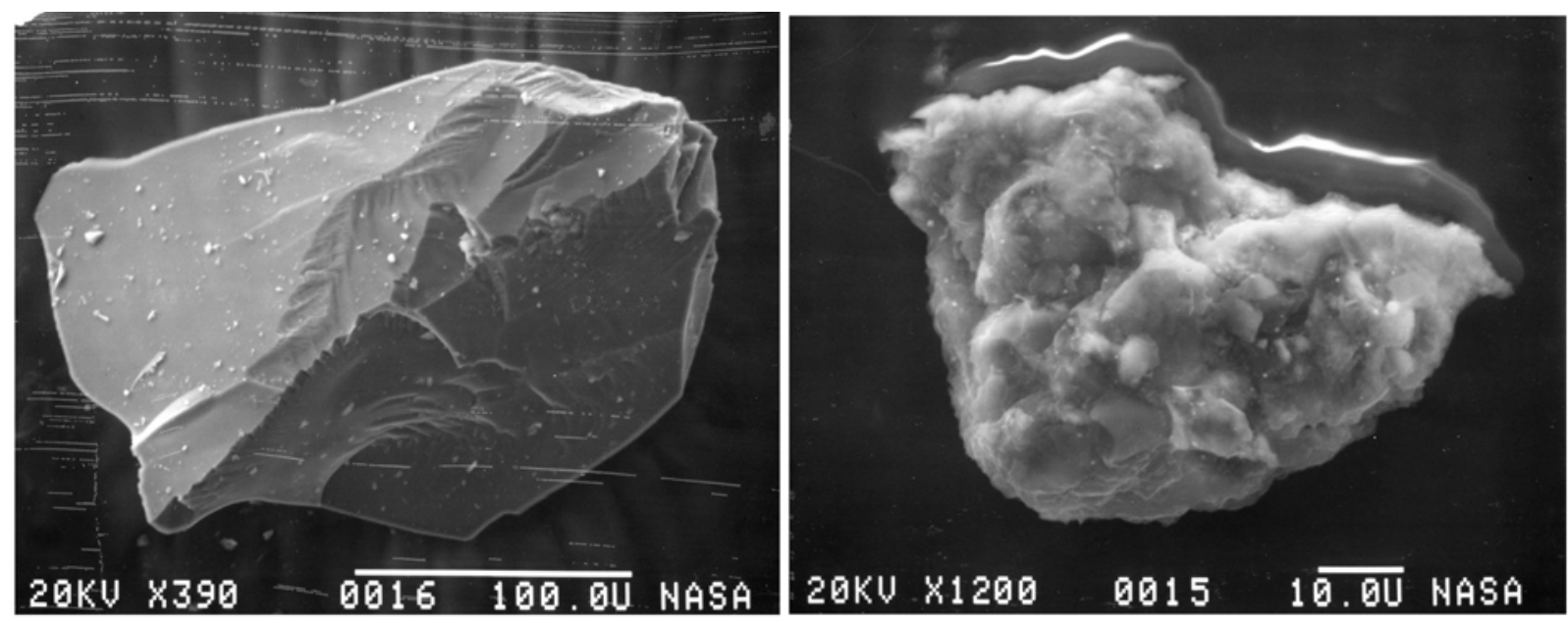

Figure 8. SEM images of a typical olivine grain before (left) and after (right) launching into aerogel in light gas gun experiments at JSC (after Barrett et al., 1993). 

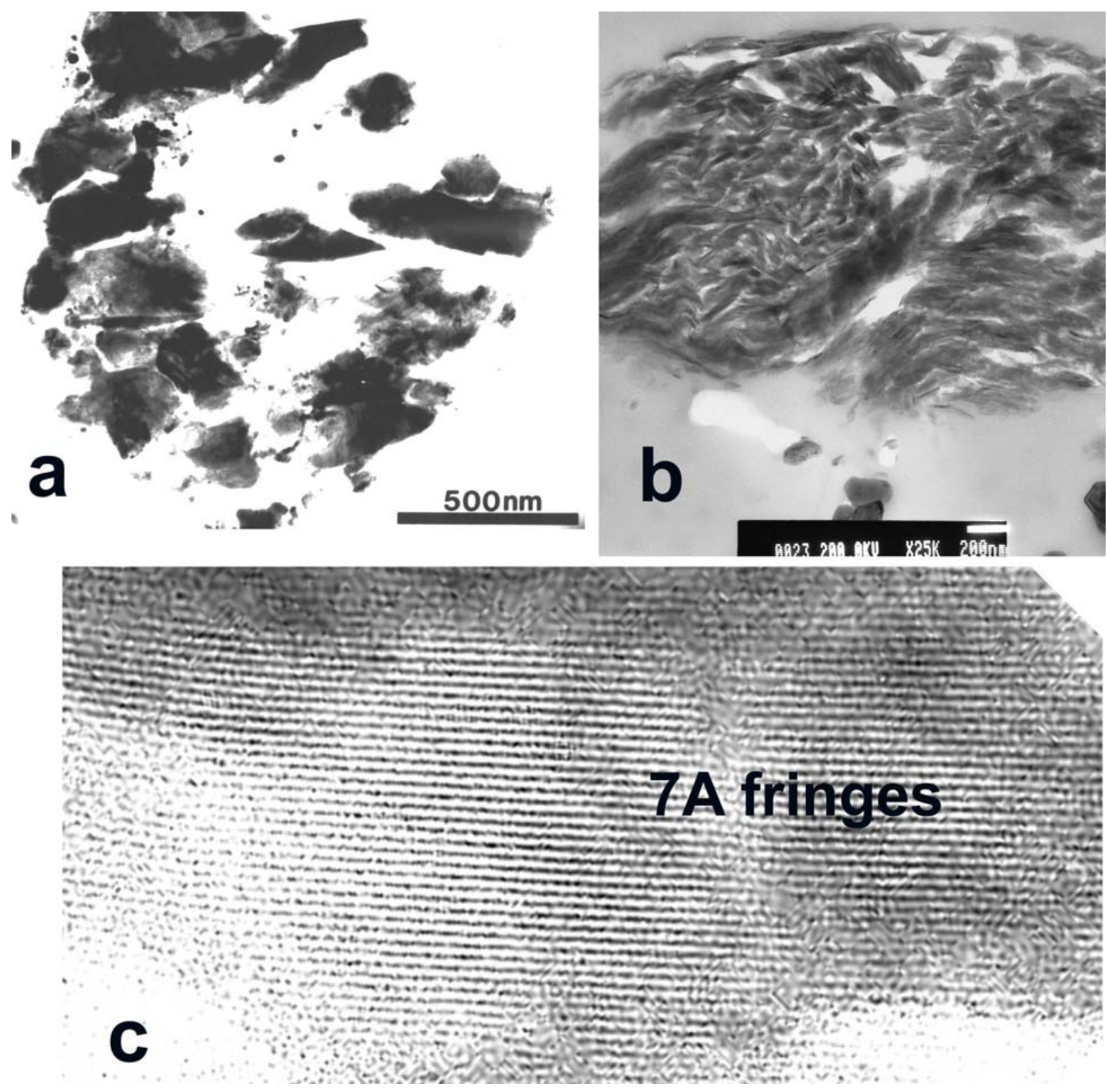

Figure 9

Figure 9. TEM images of microtome olivine grains following capture in silica aerogel at 7.2 $\mathrm{km} / \mathrm{s}$ in lab simulations. (a) Low magnification images of post-capture olivine. (b) Images of a flake of serpentine within the olivine shown in (a). (c) High-resolution TEM images of lattice fringes in the captured serpentine. The lattice spacings here are $7.4 \AA$, which is characteristic for serpentine. Elsewhere in this sample fringes are not apparent, as the structure has been modified by the capture process. However, even in these modified areas the original flaky morphology of serpentine has been preserved. 